\title{
Sitting, physical activity, and serum oestrogen metabolism in postmenopausal women: the Women's Health Initiative Observational Study
}

Hannah $\mathrm{Oh}^{\star}{ }^{* 1,2}$, Hannah Arem ${ }^{3}$, Charles E Matthews ${ }^{1}$, Nicolas Wentzensen ${ }^{1}$, Kerryn W Reding ${ }^{4}$, Louise A Brinton ${ }^{1}$, Garnet L Anderson ${ }^{5}$, Sally B Coburn ${ }^{1}$, Jane A Cauley ${ }^{6}$, Chu Chen ${ }^{5}$, Deborah Goodman ${ }^{7}$, Ruth M Pfeiffer ${ }^{1}$, Roni T Falk ${ }^{1}$, $\mathrm{Xia} \mathrm{Xu}^{8}$ and Britton Trabert ${ }^{1}$

${ }^{1}$ Division of Cancer Epidemiology and Genetics, National Cancer Institute, Bethesda, MD, USA; ${ }^{2}$ Cancer Prevention and Control, Rutgers Cancer Institute of New Jersey, New Brunswick, NJ, USA; ${ }^{3}$ Department of Epidemiology and Biostatistics, Milken Institute School of Public Health, The George Washington University, Washington, DC, USA; ${ }^{4}$ Department of Biobehavioral Nursing and Health Informatics, School of Nursing, University of Washington, Seattle, WA, USA; ${ }^{5}$ Program in Epidemiology, Division of Public Health Sciences, Fred Hutchinson Cancer Research Center, Seattle, WA, USA; ${ }^{6}$ Department of Epidemiology, Graduate School of Public Health, University of Pittsburgh, Pittsburgh, PA, USA; ${ }^{7}$ Department of Epidemiology, University of California-Irvine, Irvine, CA, USA and ${ }^{8}$ Cancer Research Technology Program, Leidos Biomedical Research, Inc., Frederick National Laboratory for Cancer Research, Frederick, MD, USA

Background: Prolonged sitting and lower levels of physical activity have been associated with increased levels of parent oestrogens (oestrone and oestradiol), the key hormones in female cancers, in postmenopausal women. However, it is unknown whether sitting and physical activity are associated with circulating oestrogen metabolite levels.

Methods: Among 1804 postmenopausal women enrolled in the Women's Health Initiative Observational Study, 15 serum oestrogens/oestrogen metabolites were quantified using liquid chromatography-tandem mass spectrometry. Physical activity and sitting were self-reported via questionnaire. Using baseline, cross-sectional data, geometric means (GM) of oestrogens/oestrogen metabolites (pmoll ${ }^{-1}$ ) were estimated using inverse probability weighted linear regression, adjusting for potential confounders and stratified on menopausal hormone therapy (MHT) use.

Results: Longer time spent sitting ( $\geqslant 10 \mathrm{vs} \leqslant 5 \mathrm{~h}$ per day) was associated with higher levels of unconjugated oestrone, independent of moderateto vigorous-intensity physical activity and body mass index, among both never/former ( $G M=70.6$ vs 57.7$)$ and current $M H T$ users (GM $=242$ vs 179$)$ ( $P$-trend $\leqslant 0.03$ ). Among never/former MHT users, sitting ( $\geqslant 10 \mathrm{vs} \leqslant 5 \mathrm{~h}$ per day) was positively associated with 2 -methoxyestradiol (GM $=16.4 \mathrm{vs}$ 14.4) and 4-methoxyestradiol ( $G M=2.36$ vs 1.98) ( $P$-trend $\leqslant 0.04)$, independent of parent oestrogens. Inverse associations between moderate- to vigorous-intensity physical activity ( $\geqslant 15$ vs 0 metabolic equivalent task-hours per week) and parent oestrogens were found as expected. After adjustment for parent oestrogens, physical activity was not associated with oestrogen metabolites.

Conclusions: Our data suggest that prolonged sitting and lower moderate- to vigorous-intensity physical activity are associated with higher levels of postmenopausal oestrogens/oestrogen metabolites, the oestrogen metabolism patterns that have previously been associated with higher endometrial and breast cancer risk.

In postmenopausal women, physical activity is associated with reduced levels of oestrogens (McTiernan et al, 2006; Chan et al, 2007; Monninkhof et al, 2009; van Gils et al, 2009; Friedenreich et al,
2010; Choudhury et al, 2011), one of the key hormones implicated in the aetiology of some female cancers (e.g., endometrial, breast, and non-serous ovarian) (Key et al, 2002; Fuhrman et al, 2012; Falk et al,

*Correspondence: Dr H Oh; E-mail: hannah.oh@rutgers.edu

Received 1 April 2017; revised 17 July 2017; accepted 19 July 2017; published online 17 August 2017

(C) 2017 Cancer Research UK. All rights reserved 0007-0920/17 
2013; Brown and Hankinson, 2015; Trabert et al, 2015; Brinton et al, 2016). In a randomised controlled trial, postmenopausal women who exercised for 150-225 min per week during a 12-month period had an $18 \%$ reduction (vs $4 \%$ in the control group) in circulating oestradiol levels from baseline (Friedenreich et al, 2010). The inverse relationships between physical activity and postmenopausal endogenous oestrogens have been consistently reported in other randomised trials (McTiernan et al, 2004; Monninkhof et al, 2009) and most observational studies (Nelson et al, 1988; Cauley et al, 1989; Madigan et al, 1998; McTiernan et al, 2006; Chan et al, 2007; van Gils et al, 2009; Choudhury et al, 2011). However, beyond parent oestrogens (oestradiol and oestrone), little is known about the association between physical activity and oestrogen metabolism. It is also unclear whether the relationship can be explained by body weight. Independent of physical activity, sedentary time has been shown to be positively associated with urinary levels of parent oestrogens (Dallal et al, 2016). To the best of our knowledge, no study to date has examined the association of sedentary time with serum levels of endogenous oestrogens in postmenopausal women. Although the exact relationships between urinary and serum oestrogens are unclear, serum levels are thought to more closely represent circulating $v s$ excreted levels of oestrogens, which may be more relevant to cancer risk. Further, while current menopausal hormone therapy (MHT) users have higher circulating oestrogen levels (Nachtigall et al, 2000; Edlefsen et al, 2010), it is unknown whether sedentary time and physical activity are associated with further differences in circulating levels in this group.

Parent oestrogens are metabolised into an array of oestrogen metabolites when hydroxylated at the 2-, 4-, or 16-carbon position. Parent oestrogens and some oestrogen metabolites stimulate cell proliferation by binding oestrogen receptors (Santen et al, 2015). Evidence has shown that certain oestrogen metabolites, namely 2- and 4-pathway catechols, can also damage DNA directly by forming quinone DNA adduct and indirectly via redox cycling (Cavalieri et al, 2000, 2006). When catechols are methylated, they do not undergo further redox cycling thus mutagenic potentials may be reduced (Yager, 2000). The degree of oestrogenic and mutagenic potentials of individual oestrogen metabolites likely depends on their affinity to oestrogen receptors (Cavalieri et al, 2000) and, for catechols, methylation status (Cavalieri et al, 2000; Yager, 2000). Hence, comprehensive evaluations of oestrogens/oestrogen metabolites may provide greater insight into the roles of sedentary time and physical activity in endogenous oestrogen metabolism. Using baseline, cross-sectional data from the Women's Health Initiative (WHI) Observational Study (OS), we examined whether sedentary time, specifically sitting, and physical activity were associated with serum levels of 15 oestrogens/oestrogen metabolites in postmenopausal women stratified by MHT usage.

\section{MATERIALS AND METHODS}

Study population. This study includes baseline, cross-sectional data from participants in a case-control study of ovarian and endometrial cancers nested within the WHI-OS (Trabert et al, 2015; Brinton et al, 2016). The WHI-OS is a prospective cohort study that recruited 93676 postmenopausal women aged 50-79 years at 40 clinical centres in the United States between 1993 and 1998 (Langer et al, 2003; The Women's Health Initiative Study Group, 1998). At baseline clinic visit, anthropometrics (e.g., height and weight) were measured and blood samples were collected. In addition, baseline self-administered questionnaires collected information on participants' demographics, medical history, and health behaviours (e.g., physical activity and sitting).

Details of the nested case-control study are described elsewhere (Trabert et al, 2015; Brinton et al, 2016). Cases were women with ovarian or endometrial cancer diagnosed between study enrolment and 2012. Participants had no history of cancer (except nonmelanoma skin cancer), bilateral oophorectomy, or hysterectomy (for endometrial controls only), and had $\geqslant 1.1 \mathrm{ml}$ serum available. Controls were selected from women who remained cancer-free at the date of case diagnosis and were frequency matched to cases based on age at baseline (5-year categories), year of blood draw (1993-1996 and 1997-1998), race/ethnicity (white, black, Hispanic, and other/unknown), hysterectomy before the index date (for ovarian controls only), and MHT use (never, $\leqslant 1$ year since last MHT use, $>1$ year since last MHT use, and current).

A total of 1804 women (492 cases and 444 controls among never/former MHT users, and 452 cases and 416 controls among current MHT users), representing 55256 women when weighted back to the entire cohort, were included in the study. Because all serum samples were collected at baseline prior to cancer diagnosis (range: 45 days-14.9 years; mean: 6.7 years), we included both cases and controls and accounted for case-control selection by weighting in this cross-sectional analysis.

Approval for conducting the study was obtained from human subjects review at the Fred Hutchinson Cancer Research Center (WHI Clinical Coordinating Center), as well as at all participating clinical centres. Written informed consent was obtained from study participants.

Exposure assessment. At baseline, levels of sitting and physical activity were self-reported via questionnaire. Sitting during a usual day (e.g., sitting at work, sitting at the table eating, driving or riding in a car or bus, and sitting up watching TV or talking) was reported in one of eight categories $(<4,4-5,6-7,8-9,10-11,12-13,14-15$, and $\geqslant 16 \mathrm{~h}$ per day). Because of sparse data, categories were collapsed into three levels: $\leqslant 5 ; 6-9$; and $\geqslant 10 \mathrm{~h}$ per day. Details of physical activity assessment have been described previously (Arem et al, 2013). Briefly, participants were asked to report their usual number of days per week $(1,2,3,4$, and $\geqslant 5)$ and minutes per session $(<20,20-39,40-59$, and $\geqslant 60)$ they spent on moderate exercise (e.g., biking outdoors and folk dancing), strenuous exercise (e.g., aerobics and swimming laps), and walking outside the home (average-paced (2-3 mph), fairly fast (3-4 mph), and very fast $(>4 \mathrm{mph})$ ). Total metabolic equivalent (MET)-hours per week of moderate- to vigorous-intensity physical activities including walking were estimated by multiplying the summed number of hours per week of each activity type with its corresponding average MET values (Ainsworth et al, 2000). According to the 2008 Physical Activity Guidelines for Americans recommended minimum ( $\geqslant 150 \mathrm{~min}$ per week of moderate-intensity exercise, equivalent to 7.5-14.9 MET-h per week) (President's Council on Fitness Sports \& Nutrition, 2016), activity levels were categorised into four levels: 0 (no exercise); 0.1-7.4 (less than the guideline); 7.5-14.9 (meeting the guideline); and $\geqslant 15$ MET-h per week (exceeding the guideline).

Laboratory assays. Details of the laboratory assays have been described previously (Trabert et al, 2015). The assay quantifies the combined concentrations of conjugated and unconjugated forms of each of the 15 oestrogens/oestrogen metabolites (oestrone, $17 \beta$ oestradiol (oestradiol), 2-hydroxyestrone, 2-hydroxyestradiol, 2methoxyestrone, 2-methoxyestradiol, 2-hydroxyestrone-3-methyl ether, 4-hydroxyestrone, 4-methoxyestrone, 4-methoxyestradiol, $16 \alpha$-hydroxyestrone, oestriol, 16-ketoestradiol, 16-epiestriol, and 17-epiestriol) and unconjugated concentrations of five analytes (oestrone, oestradiol, oestriol, 2-methoxyestrone, and 2-methoxyestradiol) in serum using a stable isotope dilution liquid chromatography-tandem mass spectrometry (LC-MS/MS) assay (Leidos Biomedical Research, Inc., Frederick, MD, USA) (Xu et al, 2007). Coefficients of variation were $<6 \%$ and intraclass correlation coefficients (ICCs) were $>0.93$ for all analytes (Trabert et al, 2015). Spearman's correlations amongst the 
oestrogens/oestrogen metabolites ranged from 0.34 to 0.99 (Brinton et al, 2016).

Statistical analyses. Because oestrogens/oestrogen metabolite levels vary by MHT usage (Nachtigall et al, 2000; Edlefsen et al, 2010), all analyses were stratified on MHT use ( $n=936$ never/ former users and 868 current users). Inverse probability sampling weights were used to adjust the data to represent the population in the entire cohort using methods for secondary phenotype analysis of case-control data described by $\mathrm{Li}$ and Gail (2012). Sampling weights were calculated by taking the inverse of sampling fractions: one for all cases and varying weights for controls depending on their strata defined by matching factors.

Distributions of serum oestrogens/oestrogen metabolite data were right-skewed; details of the data have been described elsewhere (Brinton et al, 2016). After log-transformation of data to improve normality, geometric means (GM; $\mathrm{pmoll}^{-1}$ ) of individual serum oestrogen/oestrogen metabolite concentrations by exposure categories were estimated using inverse probability weighted linear regression. All analyses were adjusted for age (5-year categories) and a priori selected potential confounders: blood draw year (1993-1996 and 1997-1998); race (white and nonwhite); smoking status (never, former, and current); and time since menopause $(<10,10-19, \geqslant 20$ years, and missing). Additional adjustment for soy and alcohol intakes and MHT formulation (among current MHT users) did not change the results, thus were not included in the final models. To account for correlated data, we evaluated models with additional adjustment for body mass index (BMI; Spearman's $r=0.12$ for sitting and -0.27 for physical activity) and mutual adjustment for sitting and physical activity (Spearman's $r=-0.11$ ). For oestrogen metabolite analyses, we assessed whether the associations were independent of associations with parent oestrogens. We performed a test for trend by including the exposure in the model as a continuous variable. We also performed cubic spline models to assess for nonlinearity of the associations; however, the model fit was not statistically significantly better than linear models $(P>0.05)$ thus are not presented. We tested for any difference in oestrogen/oestrogen metabolite levels across exposure categories using global F-test. In secondary analysis, we further investigated the relationships with patterns of oestrogen metabolism by comparing the mean proportion of parent oestrogens across exposure categories, with adjustment for the summed concentration of oestrogens/oestrogen metabolites.

Finally, to assess the influence of subclinical disease, we performed sensitivity analyses after excluding endometrial and ovarian cancer cases $(n=492$ never/former and 452 current MHT users) and excluding women with low BMI $\left(<18.5 \mathrm{~kg} \mathrm{~m}^{-2} ; n=12\right.$ never/former and 13 current MHT users). We also excluded women with a history of diabetes at baseline $(n=56$ never/former and 28 current MHT users) because diabetes and diabetic medications (e.g., metformin) may influence oestrogen levels via biologic crosstalk between insulin signalling and sex hormones. We also repeated analyses after excluding outliers ( $\leqslant 10$ values for never/former and $\leqslant 10$ values for current MHT users) identified using the extreme Studentized deviate many-outlier procedure (Rosner, 1983) and further stratifying by never $v s$ former MHT users $(n=629$ never and 307 former users). All statistical tests were two-sided with 5\% type I error. Q-values reflecting the false discovery rates (FDR) were calculated to account for multiple comparisons. Analyses were conducted with SAS version 9 (SAS Institute Inc., Cary, NC, USA).

\section{RESULTS}

Study population characteristics. The mean age was 64.4 years for never/former MHT users and 60.1 years for current MHT users. Among both never/former and current MHT users, women who reported sitting $\geqslant 10 \mathrm{~h}$ per day ( $v s \leqslant 5 \mathrm{~h}$ per day) and women reporting 0 MET-h per week ( $v s \geqslant 15$ MET-h per week) moderateto vigorous-intensity physical activity were more likely to have BMI $\geqslant 30 \mathrm{~kg} \mathrm{~m}^{-2}$ (Table 1). As expected, serum concentrations of all evaluated oestrogens/oestrogen metabolites were higher in current MHT users compared to never/former users (Table 2-4; Supplementary Tables 1-2).

Parent oestrogens. Adjusting for age and potential confounders, sitting at least for $10 \mathrm{~h}$ per day compared with sitting $\leqslant 5 \mathrm{~h}$ per day was positively associated with serum levels of unconjugated oestrone $\left(\mathrm{GM}=70.0\right.$ vs $\left.54.6 \mathrm{pmoll}^{-1}\right)$ and unconjugated oestradiol $\left(\mathrm{GM}=16.4\right.$ vs $\left.12.5 \mathrm{pmoll}^{-1}\right)$ with significant linear trends $(P$-trend $\leqslant 0.04)$ among never/former MHT users (Table 2$)$. The positive association with unconjugated oestrone remained after adjustment for BMI and physical activity $(\mathrm{GM}=70.6 \mathrm{vs}$ $57.7 \mathrm{pmoll}^{-1}, P$-trend $=0.02$ ). Among current MHT users, a similar positive association with unconjugated oestrone was observed after adjustment for BMI and physical activity $\left(\mathrm{GM}=242\right.$ vs $179 \mathrm{pmoll}^{-1}, P$-trend $\left.=0.03\right)$. Among these current MHT users, percentages of parent oestrogens out of the total measured oestrogens/oestrogen metabolites concentrations were also significantly greater in women with longer sitting hours $(\geqslant 10$ $v s \leqslant 5 \mathrm{~h}$ per day; $58 \%$ vs $53 \%, P=0.01$ ); we observed no difference in percentages among never/former MHT users ( $48 \%$ vs $49 \%$, $P=0.43$; data not tabled).

Among never/former MHT users, women who reported at least 15 MET-h per week moderate- to vigorous-intensity physical activity in comparison to women who reported no physical activity (0 MET-h per week) were associated with lower levels of oestrone $\left(\mathrm{GM}=289\right.$ vs $\left.403 \mathrm{pmoll}^{-1}\right)$ and oestradiol $(\mathrm{GM}=49.7$ vs $72.5 \mathrm{pmoll}^{-1}$ ) adjusting for age and potential confounders (all $P<0.001$; Table 2). The associations appeared to be driven by the differences between women who reported some $v s$ no physical activity ( $\geqslant 0.1$ vs 0 MET-h per week). The associations remained after adjustment for BMI and sitting $\left(\mathrm{GM}=332\right.$ vs $370 \mathrm{pmoll}^{-1}$ for oestrone; $56.2 v s 67.4 \mathrm{pmoll}^{-1}$ for oestradiol; all $P \leqslant 0.02$ ). No association between physical activity and parent oestrogens was observed among current MHT users.

Oestrogen metabolites. Among never/former MHT users, hours spent sitting $(\geqslant 10 v s \leqslant 5 \mathrm{~h}$ per day) was positively associated with 2-methoxyestradiol $\left(\mathrm{GM}=15.8\right.$ vs $12.6 \mathrm{pmoll}^{-1}, P$-trend $\left.=0.03\right)$ and 4-methoxyestradiol $\left(\mathrm{GM}=2.27\right.$ vs $1.75 \mathrm{pmoll}^{-1}$, $P$-trend $=0.02$ ) adjusting for age, potential confounders, and BMI (Table 3); the associations persisted after adjustment for parent oestrogens $\left(\mathrm{GM}=16.4\right.$ vs $14.4 \mathrm{pmoll}^{-1}, P$-trend $=0.04$; and 2.36 vs $1.98 \mathrm{pmoll}^{-1}, P$-trend $=0.03$, respectively; Table 3 ). Among current MHT users, none of the oestrogen metabolites were associated with time spent sitting (Supplementary Table 1).

Among never/former MHT users, most serum oestrogen metabolites were inversely associated with moderate- to vigorous-intensity physical activity (e.g., $\geqslant 15$ vs 0 MET-h per week: $\mathrm{GM}=66.6$ vs $80.8 \mathrm{pmoll}^{-1}$ for 2-hydroxyestrone; 16.7 vs $19.9 \mathrm{pmoll}^{-1}$ for 2-hydroxyestradiol; $8.13 v s 9.77 \mathrm{pmoll}^{-1}$ for 4hydroxyestrone; and 33.3 vs $40.1 \mathrm{pmoll}^{-1}$ for $16 \alpha$-hydroxyestrone; all $P \leqslant 0.02$ ) adjusting for age, potential confounders, and BMI (Table 4). However, after additional adjustment for parent oestrogens, these patterns did not persist. Among current MHT users, none of the oestrogen metabolites were associated with moderate- to vigorous-intensity physical activity (Supplementary Table 2).

Most parent oestrogen associations with physical activity, but not with sitting, were $<5 \%$ FDR (Tables $2-4$ ). Results were similar after excluding endometrial or ovarian cancer cases at the time of last follow-up (Supplementary Table 3), after excluding women who reported a history of diabetes at baseline (data not shown), after excluding women with low BMI $\left(<18.5 \mathrm{~kg} \mathrm{~m}^{-2}\right.$; data not 
Table 1. Characteristics of study population by sitting and moderate- to vigorous-intensity physical activity in postmenopausal women not using menopausal hormone therapy, stratified by menopausal hormone therapy use: the Women's Health Initiative Observational Study

\begin{tabular}{|c|c|c|c|c|c|c|c|}
\hline \multicolumn{8}{|c|}{ Postmenopausal women not using menopausal hormone therapy $(N=936$, weighted $N=30405)$} \\
\hline \multirow[b]{3}{*}{ Characteristic } & \multicolumn{3}{|c|}{ Sitting (h per day) } & \multicolumn{4}{|c|}{ Moderate- to vigorous-intensity physical activity (MET-h per week) } \\
\hline & $\leqslant 5$ & $6-9$ & $\geqslant 10$ & 0 & $0.1-7.4$ & $7.5-14.9$ & $\geqslant 15$ \\
\hline & \multicolumn{3}{|c|}{$N\left(\right.$ weighted $\%{ }^{a}$ ) } & \multicolumn{4}{|c|}{$N$ (weighted $\%^{a}$ ) } \\
\hline \multicolumn{8}{|c|}{ Age at baseline blood draw (years) } \\
\hline $\begin{array}{l}<55 \\
55-59 \\
60-64 \\
65-69 \\
70-74 \\
75-79\end{array}$ & $\begin{array}{l}17(4.6) \\
57(13.3) \\
71(18.6) \\
71(23.6) \\
73(28.0) \\
35(12.0)\end{array}$ & $\begin{array}{l}36(7.2) \\
63(17.9) \\
94(22.5) \\
92(27.3) \\
66(15.4) \\
38(9.7)\end{array}$ & $\begin{array}{l}27(16.9) \\
55(23.4) \\
56(21.2) \\
34(15.9) \\
29(11.9) \\
22(10.8)\end{array}$ & $\begin{array}{l}15(4.3) \\
48(22.9) \\
52(21.9) \\
37(21.8) \\
32(21.4) \\
17(7.7)\end{array}$ & $\begin{array}{l}12(8.7) \\
42(18.4) \\
51(21.6) \\
52(25.5) \\
42(18.5) \\
16(7.3)\end{array}$ & $\begin{array}{ll}20 & (8.6) \\
25 & (11.5) \\
52 & (20.7) \\
51 & (23.0) \\
41 & (18.4) \\
31 & (17.8)\end{array}$ & $\begin{array}{ll}33 & (10.5) \\
60 & (17.8) \\
66 & (19.5) \\
57 & (23.1) \\
53 & (19.4) \\
31 & (9.8)\end{array}$ \\
\hline \multicolumn{8}{|l|}{ Race } \\
\hline White & $283(89.1)$ & $342(88.0)$ & $200(91.9)$ & $164(84.2)$ & $181(84.7)$ & $200(91.1)$ & $280(94.0)$ \\
\hline \multicolumn{8}{|c|}{ Year at blood draw } \\
\hline $\begin{array}{l}1993-1996 \\
1997-1998\end{array}$ & $\begin{array}{l}188(53.6) \\
136(46.4)\end{array}$ & $\begin{array}{l}244(67.3) \\
145(32.7)\end{array}$ & $\begin{array}{r}139(64.6) \\
84(35.4)\end{array}$ & $\begin{array}{r}117(57.0) \\
84(43.0)\end{array}$ & $\begin{array}{r}131(62.4) \\
84(37.6)\end{array}$ & $\begin{array}{r}133(58.4) \\
87(41.6)\end{array}$ & $\begin{array}{l}190(66.1) \\
110(33.9)\end{array}$ \\
\hline \multicolumn{8}{|c|}{ Smoking status } \\
\hline $\begin{array}{l}\text { Never } \\
\text { Former } \\
\text { Current }\end{array}$ & $\begin{array}{c}174(56.0) \\
126(35.7) \\
24(8.3)\end{array}$ & $\begin{array}{c}190(48.5) \\
172(42.4) \\
27(9.1)\end{array}$ & $\begin{array}{c}110(43.5) \\
97(48.0) \\
16(8.5)\end{array}$ & $\begin{array}{l}92(46.5) \\
92(42.9) \\
17(10.6)\end{array}$ & $\begin{array}{c}111(52.2) \\
83(38.3) \\
21(9.5)\end{array}$ & $\begin{array}{r}117(50.9) \\
87(38.8) \\
16(10.3)\end{array}$ & $\begin{array}{c}154(50.6) \\
133(43.7) \\
13(5.7)\end{array}$ \\
\hline \multicolumn{8}{|c|}{ Time since menopause } \\
\hline $\begin{array}{l}<10 \text { years } \\
10-20 \text { years } \\
\geqslant 20 \text { years } \\
\text { Missing }\end{array}$ & $\begin{array}{c}96(27.7) \\
119(33.5) \\
100(35.4) \\
9(3.5)\end{array}$ & $\begin{array}{c}117(27.3) \\
151(41.2) \\
104(27.4) \\
17(4.1)\end{array}$ & $\begin{array}{l}83(39.0) \\
84(34.0) \\
42(20.7) \\
14(6.3)\end{array}$ & $\begin{array}{l}63(29.4) \\
83(41.9) \\
44(23.5) \\
11(5.2)\end{array}$ & $\begin{array}{c}62(29.3) \\
82(34.7) \\
66(33.5) \\
5(2.4)\end{array}$ & $\begin{array}{l}61(26.8) \\
92(38.2) \\
57(30.5) \\
10(4.5)\end{array}$ & $\begin{array}{c}110(33.4) \\
97(33.8) \\
79(27.9) \\
14(4.9)\end{array}$ \\
\hline \multicolumn{8}{|c|}{ BMI at baseline $\left(\mathrm{kg} \mathrm{m}^{-2}\right)$} \\
\hline $\begin{array}{l}<25 \\
25-30 \\
\geqslant 30\end{array}$ & $\begin{array}{r}130(45.9) \\
116(37.3) \\
78(16.8)\end{array}$ & $\begin{array}{l}141(39.5) \\
114(29.3) \\
134(31.2)\end{array}$ & $\begin{array}{l}70(47.8) \\
60(22.3) \\
93(29.9)\end{array}$ & $\begin{array}{r}38(25.8) \\
59(27.9) \\
104(46.3)\end{array}$ & $\begin{array}{l}72(41.9) \\
67(30.3) \\
76(27.8)\end{array}$ & $\begin{array}{l}78(39.9) \\
89(35.2) \\
53(24.9)\end{array}$ & $\begin{array}{r}136(54.5) \\
90(27.2) \\
74(18.4)\end{array}$ \\
\hline \multicolumn{8}{|c|}{ Postmenopausal women currently using menopausal hormone therapy $(N=868$, weighted $N=24851)$} \\
\hline \multirow[b]{3}{*}{ Characteristic } & \multicolumn{3}{|c|}{ Sitting (h per day) } & \multicolumn{4}{|c|}{ Moderate- to vigorous-intensity physical activity (MET-h per week) } \\
\hline & $\leqslant 5$ & $6-9$ & $\geqslant 10$ & 0 & $0.1-7.4$ & 7.5-14.9 & $\geqslant 15$ \\
\hline & & weighted \% & & & $N(w$ & $\left.\%^{a}\right)$ & \\
\hline \multicolumn{8}{|c|}{ Age at baseline blood draw (years) } \\
\hline $\begin{array}{l}<55 \\
55-59 \\
60-64 \\
65-69 \\
70-74 \\
75-79\end{array}$ & $\begin{array}{l}26(11.0) \\
53(24.9) \\
77(26.4) \\
72(21.7) \\
53(11.7) \\
21(4.2)\end{array}$ & $\begin{array}{l}33(15.5) \\
75(23.1) \\
88(23.1) \\
71(21.0) \\
56(11.8) \\
25(5.5)\end{array}$ & $\begin{array}{l}40(26.5) \\
65(30.9) \\
49(21.5) \\
33(9.6) \\
25(9.8) \\
6(1.8)\end{array}$ & $\begin{array}{c}16(14.0) \\
36(27.6) \\
37(27.1) \\
19(14.2) \\
29(13.1) \\
8(4.1)\end{array}$ & $\begin{array}{l}17(29.5) \\
43(24.8) \\
58(24.5) \\
38(15.0) \\
32(12.3) \\
14(4.0)\end{array}$ & $\begin{array}{l}11(6.6) \\
46(33.2) \\
44(23.2) \\
50(21.0) \\
29(12.1) \\
14(3.9)\end{array}$ & $\begin{array}{l}45(22.2) \\
68(21.7) \\
75(21.7) \\
69(21.2) \\
44(8.9) \\
16(4.2)\end{array}$ \\
\hline \multicolumn{8}{|l|}{ Race } \\
\hline White & $282(90.5)$ & $325(93.4)$ & $208(96.8)$ & $134(93.0)$ & $199(94.3)$ & $182(91.9)$ & $300(93.6)$ \\
\hline \multicolumn{8}{|c|}{ Year at blood draw } \\
\hline $\begin{array}{l}1993-1996 \\
1997-1998\end{array}$ & $\begin{array}{l}174(57.5) \\
128(42.5)\end{array}$ & $\begin{array}{l}219(63.9) \\
129(36.1)\end{array}$ & $\begin{array}{r}145(65.9) \\
73(34.1)\end{array}$ & $\begin{array}{l}90(56.1) \\
55(43.9)\end{array}$ & $\begin{array}{r}126(61.3) \\
86(38.7)\end{array}$ & $\begin{array}{r}117(54.8) \\
77(45.2)\end{array}$ & $\begin{array}{l}205(70.5) \\
112(29.5)\end{array}$ \\
\hline \multicolumn{8}{|c|}{ Smoking status } \\
\hline $\begin{array}{l}\text { Never } \\
\text { Former } \\
\text { Current }\end{array}$ & $\begin{array}{c}153(45.0) \\
137(48.3) \\
12(6.6)\end{array}$ & $\begin{array}{c}176(45.9) \\
159(50.7) \\
13(3.4)\end{array}$ & $\begin{array}{r}92(47.8) \\
116(45.2) \\
10(6.9)\end{array}$ & $\begin{array}{l}72(47.2) \\
62(40.7) \\
11(12.1)\end{array}$ & $\begin{array}{r}109(50.4) \\
95(44.3) \\
8(5.3)\end{array}$ & $\begin{array}{c}99(55.3) \\
89(40.8) \\
6(3.9)\end{array}$ & $\begin{array}{c}141(37.4) \\
166(59.9) \\
10(2.8)\end{array}$ \\
\hline \multicolumn{8}{|c|}{ Time since menopause (years) } \\
\hline $\begin{array}{l}<10 \\
10-20 \\
\geqslant 20\end{array}$ & $\begin{array}{r}98(40.5) \\
127(35.9) \\
77(23.6)\end{array}$ & $\begin{array}{r}132(38.1) \\
133(39.8) \\
83(22.1)\end{array}$ & $\begin{array}{r}115(58.5) \\
66(25.9) \\
37(15.6)\end{array}$ & $\begin{array}{l}54(38.8) \\
48(32.1) \\
43(29.1)\end{array}$ & $\begin{array}{l}84(48.0) \\
78(31.4) \\
50(20.5)\end{array}$ & $\begin{array}{l}62(36.3) \\
84(44.9) \\
48(18.8)\end{array}$ & $\begin{array}{r}145(48.9) \\
116(33.4) \\
56(17.8)\end{array}$ \\
\hline \multicolumn{8}{|c|}{ BMI at baseline $\left(\mathrm{kg} \mathrm{m}^{-2}\right)$} \\
\hline $\begin{array}{l}<25 \\
25-30 \\
\geqslant 30\end{array}$ & $\begin{array}{r}166(49.3) \\
91(32.3) \\
45(18.1)\end{array}$ & $\begin{array}{r}177(50.8) \\
96(27.7) \\
75(21.5)\end{array}$ & $\begin{array}{l}98(33.5) \\
68(36.4) \\
52(30.2)\end{array}$ & $\begin{array}{l}45(35.3) \\
47(28.1) \\
53(36.6)\end{array}$ & $\begin{array}{l}63(29.4) \\
92(44.7) \\
57(25.9)\end{array}$ & $\begin{array}{l}93(41.2) \\
69(35.4) \\
32(23.4)\end{array}$ & $\begin{array}{r}206(62.7) \\
74(25.4) \\
37(11.8)\end{array}$ \\
\hline
\end{tabular}


Table 2. Geometric means (pmoll ${ }^{-1}$ ) and $95 \% \mathrm{Cl}$ of serum parent oestrogen (oestrone and oestradiol) concentrations by sitting and moderate- to vigorous-intensity physical activity in postmenopausal women, stratified by menopausal hormone therapy use: the Women's Health Initiative Observational Study

\begin{tabular}{|c|c|c|c|c|c|c|c|c|c|}
\hline \multicolumn{10}{|c|}{ Postmenopausal women not using menopausal hormone therapy $(N=936$, weighted $N=30405)$} \\
\hline & \multicolumn{4}{|c|}{ Sitting (h per day) } & \multicolumn{5}{|c|}{ Moderate- to vigorous-intensity physical activity (MET-h per week) } \\
\hline & $\leqslant 5$ & 6-9 & $\geqslant 10$ & $P$-trend ${ }^{a}$ & 0 & $0.1-7.4$ & $7.5-14.9$ & $\geqslant 15$ & p-diff ${ }^{b}$ \\
\hline$N$ & 324 & 389 & 223 & & 201 & 215 & 220 & 300 & \\
\hline \multirow[t]{2}{*}{ Weighted $N^{c}$} & 11434 & 12162 & 6808 & & 6050 & 6723 & 7443 & 10188 & \\
\hline & \multicolumn{4}{|c|}{ Model 1} & \multicolumn{5}{|c|}{ Model 1} \\
\hline $\begin{array}{l}\text { Oestrone } \\
\text { Conjugated } \\
\text { Unconjugated }\end{array}$ & $\begin{array}{c}288(247,336) \\
227(191,269) \\
54.6(48.8,61.0)\end{array}$ & $\begin{array}{c}322(271,382) \\
251(207,303) \\
61.0(53.6,69.5)\end{array}$ & $\begin{array}{c}352(282,441) \\
273(214,348) \\
70.0(59.1,83.0)\end{array}$ & $\begin{array}{l}0.08 \\
0.13 \\
0.01\end{array}$ & $\begin{array}{c}403(329,493) \\
315(251,394) \\
74.4(63.6,87.1)\end{array}$ & $\begin{array}{c}248(205,300) \\
190(153,235) \\
50.9(44.2,58.5)\end{array}$ & $\begin{array}{c}337(275,412) \\
265(213,331) \\
62.5(53.8,72.5)\end{array}$ & $\begin{array}{c}289(238,351) \\
227(183,281) \\
55.9(48.4,64.6)\end{array}$ & $\begin{array}{l}<0.001^{d} \\
<0.001^{d} \\
<0.001^{d}\end{array}$ \\
\hline $\begin{array}{l}\text { Oestradiol } \\
\text { Conjugated } \\
\text { Unconjugated }\end{array}$ & $\begin{array}{l}51.4(42.8,61.7) \\
34.4(28.3,41.9) \\
12.5(10.2,15.4)\end{array}$ & $\begin{array}{l}56.9(47.0,68.8) \\
36.8(29.9,45.2) \\
15.6(12.7,19.2)\end{array}$ & $\begin{array}{l}63.8(50.0,81.5) \\
42.0(32.3,54.6) \\
16.4(12.8,21.0)\end{array}$ & $\begin{array}{l}0.08 \\
0.13 \\
0.04\end{array}$ & $\begin{array}{l}72.5(58.4,89.9) \\
48.2(38.5,60.4) \\
19.4(15.2,24.8)\end{array}$ & $\begin{array}{l}46.7(38.7,56.4) \\
31.2(25.1,38.8) \\
11.5(9.32,14.1)\end{array}$ & $\begin{array}{l}58.7(46.8,73.7) \\
37.1(29.2,47.3) \\
16.0(12.6,20.3)\end{array}$ & $\begin{array}{l}49.7(40.5,61.0) \\
33.2(26.7,41.3) \\
12.4(9.85,15.6)\end{array}$ & $\begin{array}{r}<0.001^{d} \\
0.002^{d} \\
<0.001^{d}\end{array}$ \\
\hline Unconjugated & \multicolumn{4}{|c|}{ Model $1+\mathrm{BMl}^{\mathrm{e}}$} & \multicolumn{5}{|c|}{ Model $1+\mathrm{BMI}^{\mathrm{e}}$} \\
\hline $\begin{array}{l}\text { Oestrone } \\
\text { Conjugated } \\
\text { Unconjugated }\end{array}$ & $\begin{array}{c}313(269,365) \\
248(208,295) \\
57.8(52.0,64.2)\end{array}$ & $\begin{array}{l}326(276,385) \\
254(211,307) \\
61.6(54.6,69.5)\end{array}$ & $\begin{array}{c}365(294,454) \\
284(223,361) \\
71.8(61.2,84.1)\end{array}$ & $\begin{array}{l}0.16 \\
0.24 \\
0.02\end{array}$ & $\begin{array}{c}367(301,448) \\
284(227,356) \\
69.9(60.5,80.8)\end{array}$ & $\begin{array}{c}264(220,318) \\
204(166,250) \\
53.2(46.4,60.9)\end{array}$ & $\begin{array}{c}362(298,441) \\
288(232,358) \\
65.7(57.0,75.6)\end{array}$ & $\begin{array}{l}328(272,397) \\
261(212,322) \\
60.9(53.0,70.0)\end{array}$ & $\begin{array}{l}0.005^{d} \\
0.008^{d} \\
0.01^{d}\end{array}$ \\
\hline $\begin{array}{l}\text { Oestradiol } \\
\text { Conjugated } \\
\text { Unconjugated }\end{array}$ & $\begin{array}{l}55.4(46.4,66.1) \\
36.8(30.3,44.6) \\
14.1(11.7,17.0)\end{array}$ & $\begin{array}{l}57.5(48.3,68.6) \\
37.2(30.6,45.1) \\
15.9(13.3,19.1)\end{array}$ & $\begin{array}{l}66.0(51.8,84.0) \\
43.3(33.3,56.2) \\
17.3(13.7,21.7)\end{array}$ & $\begin{array}{l}0.15 \\
0.20 \\
0.10\end{array}$ & $\begin{array}{l}66.8(54.3,82.3) \\
45.0(36.1,56.0) \\
17.0(13.6,21.2)\end{array}$ & $\begin{array}{l}49.4(41.1,59.4) \\
32.8(26.4,40.6) \\
12.6(10.4,15.2)\end{array}$ & $\begin{array}{l}62.6(50.2,78.1) \\
39.3(31.0,49.8) \\
17.8(14.2,22.3)\end{array}$ & $\begin{array}{l}55.5(45.4,67.8) \\
36.6(29.4,45.4) \\
14.9(12.1,18.3)\end{array}$ & $\begin{array}{l}0.02^{d} \\
0.05 \\
0.01^{d}\end{array}$ \\
\hline Unconjugated & \multicolumn{4}{|c|}{ Model $1+$ BMl $^{\mathbf{e}}+$ physical activity $^{f}$} & \multicolumn{5}{|c|}{ Model $1+$ BMle $^{\mathbf{e}}$ sitting $^{9}$} \\
\hline $\begin{array}{l}\text { Oestrone } \\
\text { Conjugated } \\
\text { Unconjugated }\end{array}$ & $\begin{array}{c}313(268,366) \\
248(208,296) \\
57.7(51.8,64.2)\end{array}$ & $\begin{array}{c}324(274,384) \\
253(209,305) \\
61.4(54.4,69.3)\end{array}$ & $\begin{array}{c}358(288,444) \\
278(219,353) \\
70.6(60.3,82.6)\end{array}$ & $\begin{array}{l}0.16 \\
0.24 \\
0.02\end{array}$ & $\begin{array}{c}370(303,451) \\
286(228,358) \\
70.6(61.2,81.4)\end{array}$ & $\begin{array}{c}269(223,323) \\
207(168,254) \\
54.4(47.4,62.4)\end{array}$ & $\begin{array}{l}365(300,444) \\
290(233,360) \\
66.4(57.7,76.3)\end{array}$ & $\begin{array}{l}332(274,402) \\
264(213,327) \\
61.8(53.7,71.0)\end{array}$ & $\begin{array}{l}0.007^{d} \\
0.01^{d} \\
0.01^{d}\end{array}$ \\
\hline $\begin{array}{l}\text { Oestradiol } \\
\text { Conjugated } \\
\text { Unconjugated }\end{array}$ & $\begin{array}{l}55.1(46.2,65.7) \\
36.6(30.2,44.4) \\
14.0(11.6,17.0)\end{array}$ & $\begin{array}{l}57.4(48.2,68.4) \\
37.1(30.5,45.0) \\
15.9(13.2,19.0)\end{array}$ & $\begin{array}{l}64.6(50.9,82.0) \\
42.4(32.7,55.0) \\
16.9(13.5,21.2)\end{array}$ & $\begin{array}{l}0.16 \\
0.21 \\
0.10\end{array}$ & $\begin{array}{l}67.4(54.5,83.2) \\
45.3(36.3,56.6) \\
17.1(13.7,21.4)\end{array}$ & $\begin{array}{l}50.3(41.7,60.8) \\
33.4(26.8,41.6) \\
12.8(10.6,15.5)\end{array}$ & $\begin{array}{l}63.2(50.6,78.9) \\
39.6(31.2,50.3) \\
17.9(14.3,22.5)\end{array}$ & $\begin{array}{l}56.2(45.7,69.0) \\
37.1(29.7,46.4) \\
14.9(12.1,18.4)\end{array}$ & $\begin{array}{l}0.02^{\mathrm{d}} \\
0.06 \\
0.02^{\mathrm{d}}\end{array}$ \\
\hline \multicolumn{10}{|c|}{ Postmenopausal women currently using menopausal hormone therapy ( $N=868$, weighted $N=24851$ ) } \\
\hline & \multicolumn{4}{|c|}{ Sitting (h per day) } & \multicolumn{5}{|c|}{ Moderate- to vigorous-intensity physical a } \\
\hline & $\leqslant 5$ & $6-9$ & $\geqslant 10$ & $P$-trend ${ }^{\mathrm{a}}$ & 0 & $0.1-7.4$ & $7.5-14.9$ & $\geqslant 15$ & $p$-diff ${ }^{b}$ \\
\hline$N$ & 302 & 348 & 218 & & 145 & 212 & 194 & 317 & \\
\hline \multirow[t]{2}{*}{ Weighted $N^{c}$} & 8172 & 9937 & 6742 & & 4642 & 6869 & 4626 & 8715 & \\
\hline & \multicolumn{4}{|c|}{ Model 1} & \multicolumn{5}{|c|}{ Model 1} \\
\hline $\begin{array}{l}\text { Oestrone } \\
\text { Conjugated } \\
\text { Unconjugated }\end{array}$ & $\begin{array}{c}2625(2007,3432) \\
2373(1803,3122) \\
183(148,226)\end{array}$ & $\begin{array}{c}3608(2697,4825) \\
3375(2505,4549) \\
252(203,311)\end{array}$ & $\begin{array}{c}3363(2468,4582) \\
3022(2193,4164) \\
232(184,294)\end{array}$ & $\begin{array}{l}0.09 \\
0.13 \\
0.08\end{array}$ & $\begin{array}{c}2832(2070,3875) \\
2571(1858,3556) \\
196(154,249)\end{array}$ & $\begin{array}{c}3051(2164,4302) \\
2769(1940,3952) \\
223(174,285)\end{array}$ & $\begin{array}{c}3460(2406,4974) \\
3253(2240,4726) \\
235(176,313)\end{array}$ & $\begin{array}{c}3190(2366,4301) \\
2912(2140,3962) \\
218(173,274)\end{array}$ & $\begin{array}{l}0.74 \\
0.65 \\
0.62\end{array}$ \\
\hline $\begin{array}{l}\text { Oestradiol } \\
\text { Conjugated } \\
\text { Unconjugated }\end{array}$ & $\begin{array}{c}370(287,476) \\
302(228,398) \\
38.8(30.9,48.6)\end{array}$ & $\begin{array}{c}484(359,653) \\
415(299,575) \\
48.6(38.7,61.0)\end{array}$ & $\begin{array}{c}455(339,612) \\
370(266,514) \\
48.8(37.0,64.4)\end{array}$ & $\begin{array}{l}0.08 \\
0.15 \\
0.09\end{array}$ & $\begin{array}{c}397(293,537) \\
324(231,455) \\
42.3(33.1,54.1)\end{array}$ & $\begin{array}{c}456(329,632) \\
370(257,534) \\
48.6(37.1,63.7)\end{array}$ & $\begin{array}{c}460(330,641) \\
392(270,568) \\
48.2(37.0,63.0)\end{array}$ & $\begin{array}{c}408(305,544) \\
345(251,474) \\
40.0(31.1,51.4)\end{array}$ & $\begin{array}{l}0.71 \\
0.77 \\
0.35\end{array}$ \\
\hline Unconjugated & \multicolumn{4}{|c|}{ Model $1+$ BMI $^{\mathrm{e}}$} & \multicolumn{5}{|c|}{ Model $1+$ BMle $^{\mathbf{e}}$} \\
\hline $\begin{array}{l}\text { Oestrone } \\
\text { Conjugated } \\
\text { Unconjugated }\end{array}$ & $\begin{array}{c}2588(1982,3380) \\
2336(1778,3069) \\
179(146,221)\end{array}$ & $\begin{array}{c}3572(2673,4775) \\
3337(2476,4497) \\
248(201,306)\end{array}$ & $\begin{array}{c}3396(2488,4636) \\
3052(2210,4213) \\
235(186,297)\end{array}$ & $\begin{array}{l}0.07 \\
0.09 \\
0.05\end{array}$ & $\begin{array}{c}2859(2094,3906) \\
2597(1881,3587) \\
199(156,252)\end{array}$ & $\begin{array}{c}3058(2171,4308) \\
2771(1943,3950) \\
223(174,285)\end{array}$ & $\begin{array}{c}3428(2387,4923) \\
3220(2219,4675) \\
231(174,308)\end{array}$ & $\begin{array}{c}3118(2297,4232) \\
2842(2071,3899) \\
211(166,266)\end{array}$ & $\begin{array}{l}0.81 \\
0.73 \\
0.71\end{array}$ \\
\hline $\begin{array}{l}\text { Oestradiol } \\
\text { Conjugated } \\
\text { Unconjugated }\end{array}$ & $\begin{array}{c}362(281,467) \\
294(223,388) \\
39.5(31.4,49.7)\end{array}$ & $\begin{array}{c}477(354,644) \\
407(293,565) \\
49.2(39.0,62.1)\end{array}$ & $\begin{array}{c}462(342,622) \\
376(269,524) \\
48.3(36.5,63.9)\end{array}$ & $\begin{array}{l}0.05 \\
0.08 \\
0.18\end{array}$ & $\begin{array}{c}405(300,547) \\
332(237,466) \\
41.5(32.5,53.0)\end{array}$ & $\begin{array}{c}458(332,633) \\
371(258,533) \\
48.6(36.9,63.9)\end{array}$ & $\begin{array}{c}451(325,627) \\
383(265,554) \\
49.1(37.3,64.7)\end{array}$ & $\begin{array}{c}388(290,521) \\
326(235,451) \\
41.8(32.2,54.2)\end{array}$ & $\begin{array}{l}0.59 \\
0.70 \\
0.42\end{array}$ \\
\hline Unconjugated & \multicolumn{4}{|c|}{ Model $1+$ BMle $^{\text {e physical activity }}{ }^{f}$} & \multicolumn{5}{|c|}{ Model $1+$ BMle $^{\text {e sitting }}{ }^{9}$} \\
\hline $\begin{array}{l}\text { Oestrone } \\
\text { Conjugated } \\
\text { Unconjugated }\end{array}$ & $\begin{array}{c}2583(1969,3388) \\
2334(1766,3085) \\
179(145,222)\end{array}$ & $\begin{array}{c}3632(2699,4887) \\
3407(2507,4631) \\
251(203,311)\end{array}$ & $\begin{array}{c}3500(2531,4839) \\
3151(2250,4413) \\
242(190,308)\end{array}$ & $\begin{array}{l}0.04 \\
0.06 \\
0.03\end{array}$ & $\begin{array}{c}2819(2091,3802) \\
2555(1874,3482) \\
196(156,246)\end{array}$ & $\begin{array}{c}3133(2233,4396) \\
2822(1989,4004) \\
228(179,290)\end{array}$ & $\begin{array}{c}3585(2524,5092) \\
3365(2345,4829) \\
242(184,317)\end{array}$ & $\begin{array}{c}3319(2457,4483) \\
3023(2214,4126) \\
224(178,281)\end{array}$ & $\begin{array}{l}0.62 \\
0.54 \\
0.50\end{array}$ \\
\hline $\begin{array}{l}\text { Oestradiol } \\
\text { Conjugated } \\
\text { Unconjugated }\end{array}$ & $\begin{array}{c}365(284,470) \\
296(224,391) \\
39.8(31.7,49.8)\end{array}$ & $\begin{array}{c}480(356,648) \\
411(295,574) \\
49.7(39.4,62.7)\end{array}$ & $\begin{array}{c}470(348,634) \\
384(273,539) \\
49.5(37.2,65.7)\end{array}$ & $\begin{array}{l}0.04 \\
0.07 \\
0.17\end{array}$ & $\begin{array}{c}400(299,536) \\
328(236,455) \\
41.1(32.4,52.0)\end{array}$ & $\begin{array}{c}468(341,642) \\
377(264,538) \\
49.5(37.4,65.3)\end{array}$ & $\begin{array}{c}468(339,647) \\
397(275,573) \\
50.7(38.9,66.2)\end{array}$ & $\begin{array}{c}409(307,544) \\
344(249,474) \\
43.7(34.0,56.3)\end{array}$ & $\begin{array}{l}0.61 \\
0.71 \\
0.35\end{array}$ \\
\hline \multicolumn{10}{|c|}{ 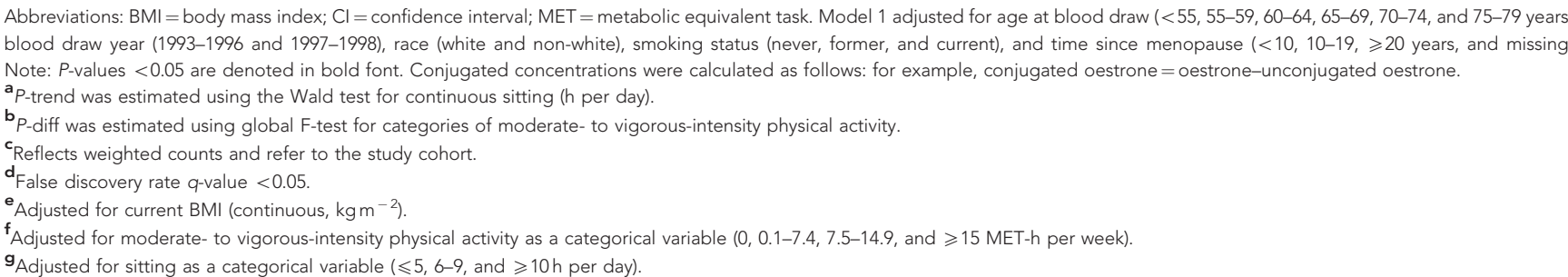 } \\
\hline
\end{tabular}


Table 3. Geometric means (pmoll ${ }^{-1}$ ) and $95 \% \mathrm{Cl}$ of serum oestrogen metabolite concentrations by sitting in postmenopausal women not using menopausal hormone therapy: the Women's Health Initiative Observational Study ( $N=936$, weighted $N=30405$ )

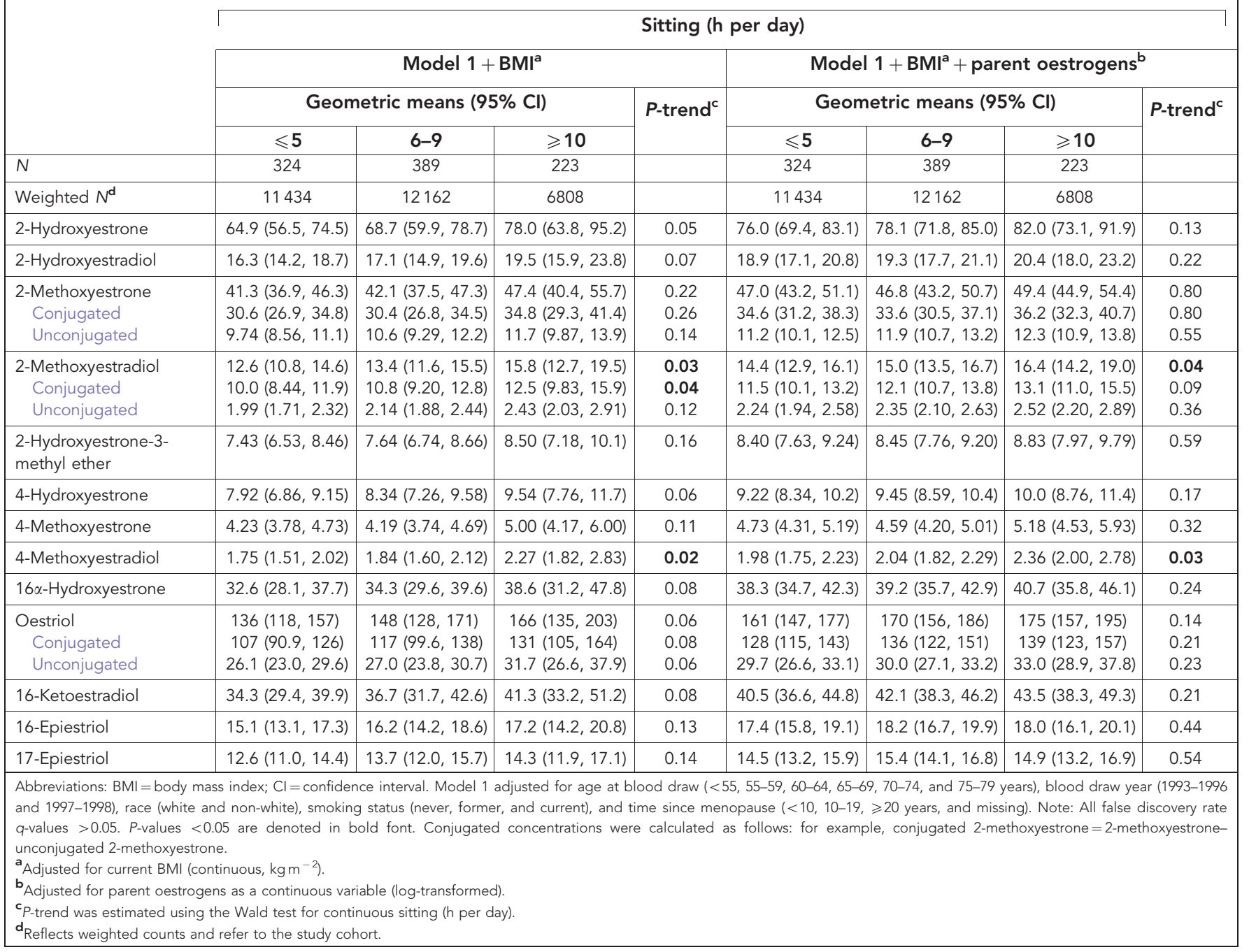

shown), and further stratifying by never $v s$ former MHT users (data not shown). Results also did not change after excluding outliers (data not shown).

\section{DISCUSSION}

To the best of our knowledge, this study is the first to examine the relationships of sitting and physical activity with detailed serum oestrogen metabolite measures in postmenopausal women stratified by MHT usage. In this cross-sectional analysis, longer hours spent sitting was associated with higher levels of unconjugated oestrone among both never/former and current MHT users, adjusted for moderate- to vigorous-intensity physical activity and current BMI. Moderate- to vigorous-intensity physical activity was inversely associated with serum levels of parent oestrogens, independent of current BMI and sitting, among never/former MHT users. Whereas physical activity was not associated with individual oestrogen metabolites after adjustment for the parent oestrogens, time spent sitting was positively associated with methylated catechols in never/former MHT users.

Although growing evidence has shown an increased endometrial (Friberg et al, 2006; Moore et al, 2010; Schmid and Leitzmann, 2014) and possibly breast (George et al, 2010; Dallal et al, 2012;
Cohen et al, 2013; Zhou et al, 2015) cancer risk associated with sitting, there are little data on sitting and oestrogens, a potential mediating pathway. A single study has evaluated urinary oestrogens in postmenopausal women not using MHT and found positive associations between accelerometer-measured sedentary time and excreted levels of oestrone and oestradiol (Dallal et al, 2016). However, no study to date has evaluated the relationships between sitting and serum oestrogens, which may be more relevant for cancer risk. The current study provides a novel finding that supports a positive association between prolonged sitting and circulating unconjugated oestrogens, the most bioavailable oestrogens, in postmenopausal women. Prolonged sitting may increase circulating oestrogens through a reduction in physical activity energy expenditure and an increase in adiposity, as adipocytes produce oestrogens via aromatase activity (Siiteri, 1987). Independent mechanisms, such as a deceleration in metabolism and excretion of oestrogens (Arciero et al, 1998; Bergouignan et al, 2011) and an alteration in lipid profiles (Healy et al, 2008, 2011; Duvivier et al, 2013; Crichton and Alkerwi, 2015), are also likely to exist because the associations with sitting persisted after adjustment for physical activity and BMI. It is also possible that the physical activity-adjusted associations were driven by other types of activity not captured in our data (e.g., light-intensity activity and occupational activity). Our data also suggest that prolonged sitting may further contribute to the differences in absolute serum 
Table 4. Geometric means (pmol/l) and $95 \%$ confidence intervals $(\mathrm{Cl})$ of serum oestrogen metabolite concentrations by moderate- to vigorous-intensity physical activity in postmenopausal women not using menopausal hormone therapy: the Women's Health Initiative Observational Study $(N=936$, weighted $N=30405)$

\begin{tabular}{|c|c|c|c|c|c|c|c|c|c|c|}
\hline & \multicolumn{10}{|c|}{ Moderate- to vigorous-intensity physical activity (MET-h per week) } \\
\hline & \multicolumn{5}{|c|}{ Model $1+$ BMI $^{a}$} & \multicolumn{5}{|c|}{ Model $1+$ BMI $^{a}+$ parent oestrogens $^{b}$} \\
\hline & \multicolumn{4}{|c|}{ Geometric means $(95 \% \mathrm{Cl})$} & \multirow[b]{2}{*}{ p-diff ${ }^{c}$} & \multicolumn{4}{|c|}{ Geometric means $(95 \% \mathrm{Cl})$} & \multirow[b]{2}{*}{ p-diff ${ }^{c}$} \\
\hline & 0 & $0.1-7.4$ & 7.5-14.9 & $\geqslant 15$ & & 0 & $0.1-7.4$ & 7.5-14.9 & $\geqslant 15$ & \\
\hline$N$ & 201 & 215 & 220 & 300 & & 201 & 215 & 220 & 300 & \\
\hline Weighted $N^{d}$ & 6050 & 6723 & 7443 & 10188 & & 6050 & 6723 & 7443 & 10188 & \\
\hline 2-Hydroxyestrone & $80.8(68.1,95.7)$ & $58.2(49.4,68.6)$ & $71.9(59.9,86.3)$ & $66.6(57.4,77.2)$ & $0.007^{e}$ & $84.1(75.4,93.7)$ & $76.5(69.0,84.8)$ & $76.1(68.9,84.2)$ & $75.7(69.1,83.0)$ & 0.25 \\
\hline 2-Hydroxyestradiol & $19.9(16.7,23.7)$ & $14.9(12.6,17.5)$ & $17.8(14.9,21.4)$ & $6.7(14.4,19.4)$ & $0.02^{\mathrm{e}}$ & $20.7(18.3,23.3)$ & $19.2(17.2,21.5)$ & $18.8(16.9,20.9)$ & $18.8(17.1,20.7)$ & 0.42 \\
\hline $\begin{array}{l}\text { 2-Methoxyestrone } \\
\text { Conjugated } \\
\text { Unconjugated }\end{array}$ & $\begin{array}{l}48.1(41.5,55.8) \\
34.6(29.3,40.8) \\
12.1(10.3,14.2)\end{array}$ & $\begin{array}{l}37.5(33.0,42.7) \\
28.3(24.5,32.6) \\
8.62(7.43,10.0)\end{array}$ & $\begin{array}{l}45.2(38.9,52.5) \\
33.1(28.2,38.8) \\
11.2(9.51,13.2)\end{array}$ & $\begin{array}{l}41.0(35.8,47.0) \\
29.7(25.5,34.6) \\
10.3(8.84,12.1)\end{array}$ & $\begin{array}{l}0.01^{e} \\
0.10 \\
0.001^{e}\end{array}$ & $\begin{array}{l}49.7(44.6,55.4) \\
35.7(31.4,40.6) \\
12.5(10.9,14.3)\end{array}$ & $\begin{array}{l}46.9(42.6,51.6) \\
35.0(31.2,39.3) \\
11.0(9.73,12.4)\end{array}$ & $\begin{array}{l}47.4(43.2,52.0) \\
34.6(31.0,38.6) \\
11.8(10.5,13.2)\end{array}$ & $\begin{array}{l}45.6(41.6,49.9) \\
32.9(29.3,36.8) \\
11.6(10.3,12.9)\end{array}$ & $\begin{array}{l}0.60 \\
0.65 \\
0.35\end{array}$ \\
\hline $\begin{array}{l}\text { 2-Methoxyestradiol } \\
\text { Conjugated } \\
\text { Unconjugated }\end{array}$ & $\begin{array}{l}15.5(12.9,18.7) \\
12.3(9.88,15.2) \\
2.48(2.10,2.94)\end{array}$ & $\begin{array}{l}11.8(10.0,13.9) \\
9.47(7.87,11.4) \\
1.80(1.54,2.09)\end{array}$ & $\begin{array}{l}14.0(11.6,16.9) \\
11.3(9.22,13.9) \\
2.23(1.89,2.62)\end{array}$ & $\begin{array}{l}13.2(11.1,15.7) \\
10.5(8.67,12.8) \\
2.09(1.81,2.42)\end{array}$ & $\begin{array}{l}0.03^{e} \\
0.07 \\
0.005^{e}\end{array}$ & $\begin{array}{l}16.1(14.0,18.5) \\
12.7(10.7,15.1) \\
2.56(2.20,2.98)\end{array}$ & $\begin{array}{l}14.9(13.2,17.0) \\
12.1(10.4,14.0) \\
2.19(1.90,2.53)\end{array}$ & $\begin{array}{l}14.7(13.1,16.5) \\
11.9(10.4,13.6) \\
2.32(2.05,2.63)\end{array}$ & $\begin{array}{l}14.7(13.0,16.7) \\
11.8(10.2,13.7) \\
2.30(2.04,2.58)\end{array}$ & $\begin{array}{l}0.62 \\
0.84 \\
0.28\end{array}$ \\
\hline $\begin{array}{l}\text { 2-Hydroxyestrone-3- } \\
\text { methyl ether }\end{array}$ & $8.43(7.20,9.85)$ & $7.04(6.13,8.08)$ & $7.81(6.67,9.14)$ & $7.72(6.71,8.90)$ & 0.17 & $8.70(7.75,9.75)$ & $8.72(7.94,9.58)$ & $8.17(7.38,9.05)$ & $8.54(7.76,9.41)$ & 0.62 \\
\hline 4-Hydroxyestrone & $9.77(8.21,11.6)$ & $7.20(6.08,8.52)$ & $8.71(7.23,10.5)$ & $8.13(6.99,9.45)$ & $0.02^{\mathrm{e}}$ & $10.2(8.98,11.5)$ & $9.38(8.34,10.5)$ & $9.21(8.25,10.3)$ & $9.21(8.31,10.2)$ & 0.39 \\
\hline 4-Methoxyestrone & $4.71(4.05,5.49)$ & $3.91(3.42,4.46)$ & $4.55(3.88,5.32)$ & $1.36(3.83,4.96)$ & 0.10 & $4.85(4.28,5.50)$ & $4.75(4.25,5.31)$ & $4.74(4.26,5.27)$ & $4.78(4.30,5.31)$ & 0.99 \\
\hline 4-Methoxyestradiol & $2.13(1.78,2.55)$ & $1.68(1.41,1.98)$ & $1.90(1.58,2.29)$ & $.90(1.62,2.22)$ & 0.08 & $2.20(1.90,2.55)$ & $2.08(1.81,2.40)$ & $1.99(1.74,2.28)$ & $2.10(1.84,2.40)$ & 0.60 \\
\hline $16 \alpha$-Hydroxyestrone & $40.1(33.5,47.9)$ & $29.0(24.4,34.6)$ & $36.0(29.7,43.7)$ & $33.3(28.5,39.0)$ & $0.01^{e}$ & $41.7(37.2,46.9)$ & $38.6(34.5,43.1)$ & $38.3(34.3,42.7)$ & $38.1(34.4,42.1)$ & 0.40 \\
\hline $\begin{array}{l}\text { Oestriol } \\
\text { Conjugated } \\
\text { Unconjugated }\end{array}$ & $\begin{array}{c}170(143,201) \\
134(110,162) \\
31.0(26.7,36.0)\end{array}$ & $\begin{array}{c}122(102,145) \\
95.6(78.8,116) \\
22.8(19.6,26.4)\end{array}$ & $\begin{array}{c}154(127,186) \\
121(98.4,149) \\
29.8(25.3,35.2)\end{array}$ & $\begin{array}{c}144(123,170) \\
116(96.7,138) \\
27.5(23.8,31.7)\end{array}$ & $\begin{array}{l}0.01^{e} \\
0.03^{e} \\
0.004^{e}\end{array}$ & $\begin{array}{c}177(159,197) \\
140(124,159) \\
32.0(28.0,36.6)\end{array}$ & $\begin{array}{c}164(148,183) \\
131(116,148) \\
28.4(25.2,32.1)\end{array}$ & $\begin{array}{c}164(148,181) \\
130(115,146) \\
31.2(27.9,35.0)\end{array}$ & $\begin{array}{c}166(150,184) \\
134(120,151) \\
30.5(27.2,34.3)\end{array}$ & $\begin{array}{l}0.57 \\
0.69 \\
0.39\end{array}$ \\
\hline 16-Ketoestradiol & $42.1(35.0,50.5)$ & $30.9(25.8,37.0)$ & $38.2(31.4,46.6)$ & $36.2(30.8,42.6)$ & $0.03^{e}$ & $43.9(39.1,49.2)$ & $41.2(36.7,46.3)$ & $40.6(36.3,45.5)$ & $41.5(37.4,46.1)$ & 0.65 \\
\hline 16-Epiestriol & $18.2(15.3,21.7)$ & $13.9(11.8,16.2)$ & $16.3(13.7,19.3)$ & $5.7(13.5,18.2)$ & $0.03^{e}$ & $18.9(16.9,21.1)$ & $17.8(16.0,19.7)$ & $17.2(15.6,19.0)$ & $17.7(16.0,19.4)$ & 0.43 \\
\hline 17-Epiestriol & $15.1(12.7,17.9)$ & $11.5(9.83,13.4)$ & $13.8(11.8,16.2)$ & $13.2(11.4,15.3)$ & $0.03^{\circ}$ & $15.7(14.0,17.6)$ & $14.7(13.1,16.4)$ & $14.5(13.2,16.0)$ & $14.8(13.5,16.3)$ & 0.58 \\
\hline \multicolumn{11}{|c|}{ 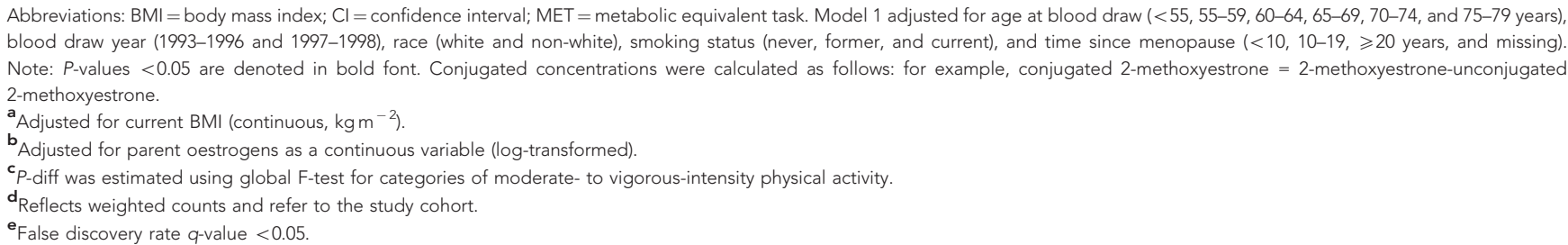 } \\
\hline
\end{tabular}

concentrations of unconjugated oestrogens among current MHT users who already have high circulating oestrogens. Among current MHT users, women with longer sitting hours also appeared to be less likely to metabolise parent oestrogens into downstream oestrogen metabolites as proportions of parent oestrogens out of the total measured oestrogens/oestrogen metabolites concentrations were higher in these women, possibly due to the saturation of cytochrome $\mathrm{P} 450$ enzymes that regulate hydroxylation of parent oestrogens to downstream metabolites.

Consistent with previous studies (McTiernan et al, 2006; Chan et al, 2007; Monninkhof et al, 2009; van Gils et al, 2009; Friedenreich et al, 2010; Choudhury et al, 2011), we observed inverse associations between physical activity and endogenous parent oestrogens among never/former MHT users. To date, three intervention trials (McTiernan et al, 2004; Monninkhof et al, 2009; Friedenreich et al, 2010) have evaluated the effect of exercise among inactive postmenopausal women not using MHT and have shown a reduction in circulating oestrogen levels as early as within 3 months (McTiernan et al, 2004). While there are limited data on current MHT users, a single observational study has reported similar inverse associations between physical activity and parent oestrogens among current MHT users (Choudhury et al, 2011); however, the associations were not observed in the current study. The exact biologic mechanisms explaining the inverse association between physical activity and parent oestrogens are not clear. Our data suggest that the associations are likely independent of BMI, as the associations remained after adjustment for current BMI. Our findings agree with all (Madigan et al, 1998; McTiernan et al, 2006; Friedenreich et al, 2010; Choudhury et al, 2011) but one (van Gils et al, 2009) previous observational studies that assessed associations adjusted for BMI. In a randomised trial (McTiernan et al, 2004), a reduction in oestrogen levels was restricted to women in the exercise arm who lost $\geqslant 2 \%$ body fat during the study period (measured by dual-energy X-ray absorptiometry), which suggests that the effects of physical activity were primarily mediated by the change in body fat. Further studies are needed to clarify the role of body fat and whether physical activity may lower oestrogen levels through other independent pathways.

Our findings show that physical activity is related to increased levels of circulating parent oestrogens but not oestrogen metabolites. Previous studies evaluated oestrogen metabolites in urine only (Atkinson et al, 2004; Dallal et al, 2016). A randomised trial examined the effect of exercise on select oestrogen metabolites, 2hydroxyestrone and $16 \alpha$-hydroxyestrone, in postmenopausal women and found no difference in the urinary excretion of these oestrogen metabolites or their ratio (Atkinson et al, 2004). A crosssectional analysis reported inverse associations of physical activity with urinary levels of methylated catechols and certain 16-pathway oestrogen metabolites (Dallal et al, 2016). However, these studies did not investigate relationships with oestrogen metabolites independent of parent oestrogens, which serve as precursors for oestrogen metabolites. In the current study, we found similar 
associations in unadjusted models; however, physical activity was not associated with serum oestrogen metabolites after adjustment for parent oestrogens. In contrast, the positive associations between sitting and methylated catechols persisted after adjustment for parent oestrogens.

We acknowledge several limitations of this study. We used a single measurement of circulating oestrogens/oestrogen metabolites at baseline. However, moderate to high 1-year ICCs reported in a previous study for most oestrogens/oestrogen metabolites (range 0.35-0.72), except for 2-methoxyestrone (0.10) and 2-hydroxyestrone-3-methyl-ether (0.14) (Falk et al, 2013), suggest that our data may adequately represent levels over at least 1 year. Although we cannot exclude the possibility of false positives, most associations with physical activity remained at 5\% FDR. Finally, physical activity and sitting were based on self-reported measures; however, measurement error in this context is unlikely to be related to serum oestrogen levels. A prior study has shown a moderate test-retest reliability $(\mathrm{ICC}=0.77$ ) for moderate- to vigorous-intensity physical activity data reported using the WHI questionnaire (Meyer et al, 2009).

Despite these limitations, this study has important strengths. Use of high-performance LC-MS/MS assay allowed comprehensive evaluations of individual oestrogens/oestrogen metabolites with high reliability, sensitivity, and specificity. By stratifying the analysis by MHT usage, we were able to take into account variations in oestrogens/oestrogen metabolite levels by exogenous hormone use. Further, use of a large sample size and careful adjustment for potential confounders assessed at blood collection increased the validity of the results.

In this comprehensive analysis of serum oestrogens/oestrogen metabolites, we observed positive associations between sitting and parent oestrogens and inverse associations between physical activity and parent oestrogens, independent of current BMI. Independent of parent oestrogens, sitting was positively associated with methylated catechols and physical activity was not associated with any of the evaluated oestrogen metabolites in postmenopausal women. These findings suggest that both sitting and physical activity are likely to influence circulating levels of parent oestrogens but only sitting was associated with oestrogen metabolites.

\section{ACKNOWLEDGEMENTS}

The WHI programme is supported by contracts from the National Heart, Lung and Blood Institute, NIH (Program Office: Jacques Rossouw; Shari Ludlam; Joan McGowan; Leslie Ford; and Nancy Geller). We thank the WHI Clinical Coordinating Center (Fred Hutchinson Cancer Research Center, Seattle WA) and the WHI investigators (Garnet Anderson, Ross Prentice, Andrea LaCroix, Charles Kooperberg, JoAnn E Manson, Barbara V Howard, Marcia L Stefanick, Rebecca Jackson, Cynthia A Thomson, Jean WactawskiWende, Marian Limacher, Jennifer Robinson, Lewis Kuller, Sally Shumaker, Robert Brunner, and Karen L Margolis) for their dedication, and the study participants for making the programme possible. A full list of WHI investigators can be found at https:// www.whi.org/researchers/Documents\%20\%20Write\%20a\%20Paper/ WHI\%20Investigator\%20Long\%20List.pdf. This work was supported by the National Heart, Lung and Blood Institute, National Institutes of Health, U.S. Department of Health and Human Services through contracts HHSN268201100046C, HHSN268201100001C, HHSN268201100002C, HHSN268201100003C HHSN268201100004C, and HHSN271201100004C. This study was also supported by the Intramural Research Program of the Division of Cancer Epidemiology and Genetics of the National Cancer Institute.

\section{CONFLICT OF INTEREST}

The authors declare no conflict of interest.

\section{REFERENCES}

Ainsworth BE, Haskell WL, Whitt MC, Irwin ML, Swartz AM, Strath SJ, O’Brien WL, Bassett Jr DR, Schmitz KH, Emplaincourt PO, Jacobs Jr DR, Leon AS (2000) Compendium of physical activities: an update of activity codes and MET intensities. Med Sci Sports Exerc 32(9 Suppl): S498-S504.

Arciero PJ, Smith DL, Calles-Escandon J (1998) Effects of short-term inactivity on glucose tolerance, energy expenditure, and blood flow in trained subjects. J Appl Physiol (1985) 84(4): 1365-1373.

Arem H, Chlebowski R, Stefanick ML, Anderson G, Wactawski-Wende J, Sims S, Gunter MJ, Irwin ML (2013) Body mass index, physical activity, and survival after endometrial cancer diagnosis: results from the Women's Health Initiative. Gynecol Oncol 128(2): 181-186.

Atkinson C, Lampe JW, Tworoger SS, Ulrich CM, Bowen D, Irwin ML, Schwartz RS, Rajan BK, Yasui Y, Potter JD (2004) Effects of a moderate intensity exercise intervention on estrogen metabolism in postmenopausal women. Cancer Epidemiol Biomarkers Prev 13(5): 868-874.

Bergouignan A, Rudwill F, Simon C, Blanc S (2011) Physical inactivity as the culprit of metabolic inflexibility: evidence from bed-rest studies. J Appl Physiol (1985) 111(4): 1201-1210.

Brinton LA, Trabert B, Anderson GL, Falk RT, Felix AS, Fuhrman BJ, Gass ML, Kuller LH, Pfeiffer RM, Rohan TE, Strickler HD, Xu X, Wentzensen N (2016) Serum estrogens and estrogen metabolites and endometrial cancer risk among postmenopausal women. Cancer Epidemiol Biomarkers Prev 25(7): 1081-1089.

Brown SB, Hankinson SE (2015) Endogenous estrogens and the risk of breast, endometrial, and ovarian cancers. Steroids 99(Pt A): 8-10.

Cauley JA, Gutai JP, Kuller LH, LeDonne D, Powell JG (1989) The epidemiology of serum sex hormones in postmenopausal women. Am J Epidemiol 129(6): 1120-1131.

Cavalieri E, Chakravarti D, Guttenplan J, Hart E, Ingle J, Jankowiak R, Muti P, Rogan E, Russo J, Santen R, Sutter T (2006) Catechol estrogen quinones as initiators of breast and other human cancers: implications for biomarkers of susceptibility and cancer prevention. Biochim Biophys Acta 1766(1): 63-78.

Cavalieri E, Frenkel K, Liehr JG, Rogan E, Roy D (2000) Estrogens as endogenous genotoxic agents - DNA adducts and mutations. J Natl Cancer Inst Monogr (27): 75-93.

Chan MF, Dowsett M, Folkerd E, Bingham S, Wareham N, Luben R, Welch A, Khaw KT (2007) Usual physical activity and endogenous sex hormones in postmenopausal women: the European prospective investigation into cancer-norfolk population study. Cancer Epidemiol Biomarkers Prev 16(5): 900-905.

Choudhury F, Bernstein L, Hodis HN, Stanczyk FZ, Mack WJ (2011) Physical activity and sex hormone levels in estradiol- and placebo-treated postmenopausal women. Menopause 18(10): 1079-1086.

Cohen SS, Matthews CE, Bradshaw PT, Lipworth L, Buchowski MS, Signorello LB, Blot WJ (2013) Sedentary behavior, physical activity, and likelihood of breast cancer among Black and White women: a report from the Southern Community Cohort Study. Cancer Prev Res 6(6): 566-576.

Crichton GE, Alkerwi A (2015) Physical activity, sedentary behavior time and lipid levels in the Observation of Cardiovascular Risk Factors in Luxembourg study. Lipids Health Dis 14: 87.

Dallal CM, Brinton LA, Matthews CE, Lissowska J, Peplonska B, Hartman TJ, Gierach GL (2012) Accelerometer-based measures of active and sedentary behavior in relation to breast cancer risk. Breast Cancer Res Treat 134(3): 1279-1290.

Dallal CM, Brinton LA, Matthews CE, Pfeiffer RM, Hartman TJ, Lissowska J, Falk RT, Garcia-Closas M, Xu X, Veenstra TD, Gierach GL (2016) Association of active and sedentary behaviors with postmenopausal estrogen metabolism. Med Sci Sports Exerc 48(3): 439-448.

Duvivier BM, Schaper NC, Bremers MA, van Crombrugge G, Menheere PP, Kars M, Savelberg HH (2013) Minimal intensity physical activity (standing and walking) of longer duration improves insulin action and plasma lipids more than shorter periods of moderate to vigorous exercise 
(cycling) in sedentary subjects when energy expenditure is comparable. PLoS One 8(2): e55542.

Edlefsen KL, Jackson RD, Prentice RL, Janssen I, Rajkovic A, O'Sullivan MJ, Anderson G (2010) The effects of postmenopausal hormone therapy on serum estrogen, progesterone, and sex hormone-binding globulin levels in healthy postmenopausal women. Menopause 17(3): 622-629.

Falk RT, Brinton LA, Dorgan JF, Fuhrman BJ, Veenstra TD, Xu X, Gierach GL (2013) Relationship of serum estrogens and estrogen metabolites to postmenopausal breast cancer risk: a nested case-control study. Breast Cancer Res 15(2): R34.

Friberg E, Mantzoros CS, Wolk A (2006) Physical activity and risk of endometrial cancer: a population-based prospective cohort study. Cancer Epidemiol Biomarkers Prev 15(11): 2136-2140.

Friedenreich CM, Woolcott CG, McTiernan A, Ballard-Barbash R, Brant RF, Stanczyk FZ, Terry T, Boyd NF, Yaffe MJ, Irwin ML, Jones CA, Yasui Y, Campbell KL, McNeely ML, Karvinen KH, Wang Q, Courneya KS (2010) Alberta physical activity and breast cancer prevention trial: sex hormone changes in a year-long exercise intervention among postmenopausal women. J Clin Oncol 28(9): 1458-1466.

Fuhrman BJ, Schairer C, Gail MH, Boyd-Morin J, Xu X, Sue LY, Buys SS, Isaacs C, Keefer LK, Veenstra TD, Berg CD, Hoover RN, Ziegler RG (2012) Estrogen metabolism and risk of breast cancer in postmenopausal women. J Natl Cancer Inst 104(4): 326-339.

George SM, Irwin ML, Matthews CE, Mayne ST, Gail MH, Moore SC, Albanes D, Ballard-Barbash R, Hollenbeck AR, Schatzkin A, Leitzmann MF (2010) Beyond recreational physical activity: examining occupational and household activity, transportation activity, and sedentary behavior in relation to postmenopausal breast cancer risk. Am J Public Health 100(11): 2288-2295.

Healy GN, Matthews CE, Dunstan DW, Winkler EA, Owen N (2011) Sedentary time and cardio-metabolic biomarkers in US adults: NHANES 2003-06. Eur Heart J 32(5): 590-597.

Healy GN, Wijndaele K, Dunstan DW, Shaw JE, Salmon J, Zimmet PZ, Owen N (2008) Objectively measured sedentary time, physical activity, and metabolic risk: the Australian Diabetes, Obesity and Lifestyle Study (AusDiab). Diabetes Care 31(2): 369-371.

Key T, Appleby P, Barnes I, Reeves G, Endogenous H. Breast Cancer Collaborative G (2002) Endogenous sex hormones and breast cancer in postmenopausal women: reanalysis of nine prospective studies. $J$ Natl Cancer Inst 94(8): 606-616.

Langer RD, White E, Lewis CE, Kotchen JM, Hendrix SL, Trevisan M (2003) The Women's Health Initiative Observational Study: baseline characteristics of participants and reliability of baseline measures. Ann Epidemiol 13(9 Suppl): S107-S121.

Li H, Gail MH (2012) Efficient adaptively weighted analysis of secondary phenotypes in case-control genome-wide association studies. Hum Hered 73(3): 159-173.

Madigan MP, Troisi R, Potischman N, Dorgan JF, Brinton LA, Hoover RN (1998) Serum hormone levels in relation to reproductive and lifestyle factors in postmenopausal women (United States). Cancer Causes Control 9(2): 199-207.

McTiernan A, Tworoger SS, Ulrich CM, Yasui Y, Irwin ML, Rajan KB, Sorensen B, Rudolph RE, Bowen D, Stanczyk FZ, Potter JD, Schwartz RS (2004) Effect of exercise on serum estrogens in postmenopausal women: a 12-month randomized clinical trial. Cancer Res 64(8): 2923-2928.

McTiernan A, Wu L, Chen C, Chlebowski R, Mossavar-Rahmani Y, Modugno F, Perri MG, Stanczyk FZ, Van Horn L, Wang CY. Women's Health Initiative I (2006) Relation of BMI and physical activity to sex hormones in postmenopausal women. Obesity 14(9): 1662-1677.
Meyer AM, Evenson KR, Morimoto L, Siscovick D, White E (2009) Test-retest reliability of the Women's Health Initiative physical activity questionnaire. Med Sci Sports Exerc 41(3): 530-538.

Monninkhof EM, Velthuis MJ, Peeters PH, Twisk JW, Schuit AJ (2009) Effect of exercise on postmenopausal sex hormone levels and role of body fat: a randomized controlled trial. J Clin Oncol 27(27): 4492-4499.

Moore SC, Gierach GL, Schatzkin A, Matthews CE (2010) Physical activity, sedentary behaviours, and the prevention of endometrial cancer. $\mathrm{Br} \mathrm{J}$ Cancer 103(7): 933-938.

Nachtigall LE, Raju U, Banerjee S, Wan L, Levitz M (2000) Serum estradiol-binding profiles in postmenopausal women undergoing three common estrogen replacement therapies: associations with sex hormone-binding globulin, estradiol, and estrone levels. Menopause 7(4): 243-250.

Nelson ME, Meredith CN, Dawson-Hughes B, Evans WJ (1988) Hormone and bone mineral status in endurance-trained and sedentary postmenopausal women. J Clin Endocrinol Metabol 66(5): 927-933.

President's Council on Fitness Sports \& Nutrition (2016) Physical Activity Guidelines for Americans Vol. 2016. Available at: http://www.fitness.gov/ be-active/physical-activity-guidelines-for-americans/.

Rosner B (1983) Percentage points for a generalized ESD many-outlier procedure. Technometrics 25(2): 165-172.

Santen RJ, Yue W, Wang JP (2015) Estrogen metabolites and breast cancer. Steroids 99(Pt A): 61-66.

Schmid D, Leitzmann MF (2014) Television viewing and time spent sedentary in relation to cancer risk: a meta-analysis. J Natl Cancer Inst 106(7): dju098.

Siiteri PK (1987) Adipose tissue as a source of hormones. Am J Clin Nutr 45(1 Suppl): 277-282.

The Women's Health Initiative Study Group (1998) Design of the Women's Health Initiative clinical trial and observational study. Control Clin Trials 19(1): 61-109.

Trabert B, Brinton LA, Anderson GL, Pfeiffer R, Falk RT, Strickler HD, Sliesoraitis S, Kuller LH, Gass ML, Fuhrman BJ, Xu X, Wentzensen N (2015) Circulating estrogens and postmenopausal ovarian cancer risk in the Women's Health Initiative Observational Study. Cancer Epidemiol Biomarkers Prev 25(4): 648-656.

van Gils CH, Peeters PH, Schoenmakers MC, Nijmeijer RM, Onland-Moret NC, van der Schouw YT, Monninkhof EM (2009) Physical activity and endogenous sex hormone levels in postmenopausal women: a crosssectional study in the Prospect-EPIC Cohort. Cancer Epidemiol Biomarkers Prev 18(2): 377-383.

Xu X, Roman JM, Issaq HJ, Keefer LK, Veenstra TD, Ziegler RG. Quantitative measurement of endogenous estrogens and estrogen metabolites in human serum by liquid chromatography-tandem mass spectrometry. Anal Chem 2007; 79: 7813-7821.

Yager JD (2000) Endogenous estrogens as carcinogens through metabolic activation. J Natl Cancer Inst Monogr (27): 67-73.

Zhou Y, Zhao H, Peng C (2015) Association of sedentary behavior with the risk of breast cancer in women: update meta-analysis of observational studies. Ann Epidemiol 25(9): 687-697.

This work is published under the standard license to publish agreement. After 12 months the work will become freely available and the license terms will switch to a Creative Commons AttributionNonCommercial-Share Alike 4.0 Unported License.

Supplementary Information accompanies this paper on British Journal of Cancer website (http://www.nature.com/bjc) 